\title{
Correction: Protease Nexin I is a feedback regulator of EGF/ PKC/MAPK/EGR1 signaling in breast cancer cells metastasis and stemness
}

Tingting Tang, Qinhua Zhu, Xinping Li, Gaole Zhu, Siwei Deng, Yingshan Wang, Lingyu Ni, Xinyuan Chen, Yanfeng Zhang, Tiansong Xia, Ke Zen $\mathbb{B}$, Yi Pan and Liang Jin

\section{Correction to: Cell Death and Disease (2019) https://doi.org/10.1038/s41419-019-1882-9, published online 9 September 2019.}

Since online publication of this article, the authors noticed that there was an error in the images used to compile Figs. 2 and 7. An incorrect MCF-7 spheroid GAPDH image was used in Fig. 2c, and incorrect MCF-7 and MCF-7 spheroid flow charts were used in Fig. 2e. In addition, an incorrect image was used for the Day 20
PN-1 lentivirus BLI mouse image for Fig. 7c. The corrected images are provided below. The authors confirm that these errors do not affect the results and conclusions of the study.

This has been corrected in the PDF and HTML versions of the article.

The authors apologize for any inconvenience caused.

Published online: 06 January 2020 


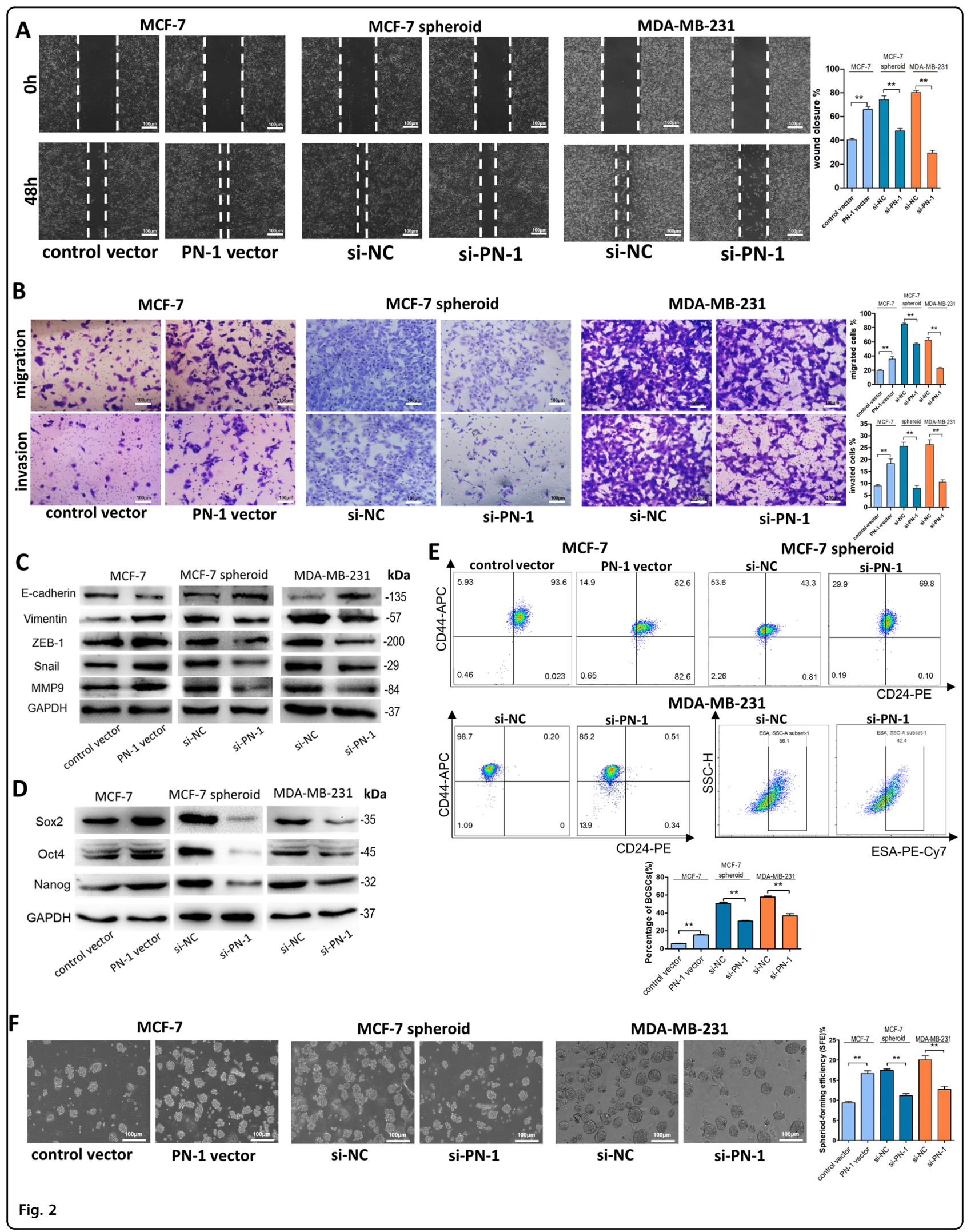




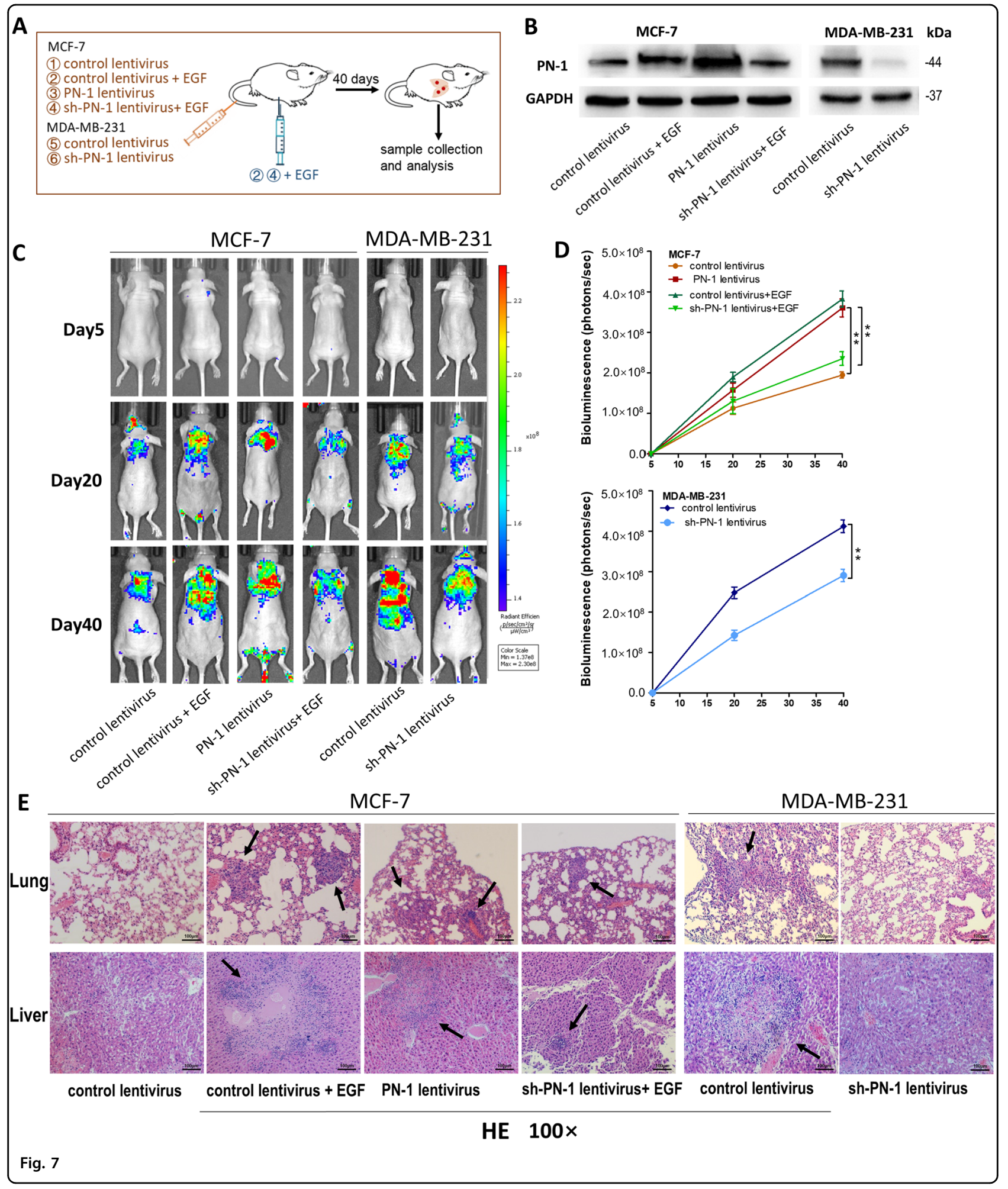

